\title{
Migrated Disc in the Lumbar Spinal Canal
}

- Case Report-

\author{
Takuya AKAI, Hideaki IIZUKA, Kazuya OKAMOTO, and Masaru KATO
}

Department of Neurosurgery, Kanazawa Medical University, Ishikawa

\begin{abstract}
A 49-year-old man who had complained of back pain for 20 years presented with numbness and pain in his left leg persisting for 6 weeks. Magnetic resonance imaging demonstrated a peripherally enhanced intraspinal mass at the L-3 level. The mass was completely removed. The operative and histological findings revealed degenerated disc fragments surrounded by granulation tissue. His symptoms were completely relieved. Migrated disc should be included in the differential diagnosis of patients with a long history of back pain and an intraspinal mass.
\end{abstract}

Key words: disc migration, lumbar spine

\section{Introduction}

Herniated disc is one of the most common causes of lumbar radiculopathy. Sequestration of the herniated disc is not uncommon. Computed tomography (CT) and magnetic resonance (MR) imaging are useful to identify the sequestered disc, ${ }^{5,9)}$ but the sequestered disc is difficult to distinguish from neoplasm, when the disc has migrated away from the intervertebral space and/or the intervertebral disc undergoes no degeneration. We report a case of migrated disc which was difficult to identify preoperatively.

\section{Case Report}

A 49-year-old man had complained of low back pain for 20 years. He suffered numbness and pain in his left leg for 6 weeks. He then visited a local clinic. MR imaging detected a mass lesion in the lumbar spinal canal. The symptom was localized at the left L-3 territory at onset, but extended to the left L-4 territory. He was referred to our institution for further evaluation and treatment.

Neurological examination found hypesthesia and paresthesia in the left L-3 and L-4 nerve dermatomes. No motor weakness was observed. Roentgenography revealed no abnormality. Myelography revealed a filling defect at L-3 vertebral body level

Received December 7, 1998; Accepted May 19, 1999 and mild compression of the subarachnoid space at the L4-5 intervertebral level (Fig. 1A, B). CT after myelography revealed an intraspinal epidural mass at the dorsal edge of the L-3 vertebral body, but no bone erosion was observed (Fig. 1C). MR imaging revealed a peripherally enhanced mass at the dorsal edge of the L-3 vertebral body. Mild protrusion of the L4-5 intervertebral disc was also observed, but no other remarkable disc degeneration was found (Fig. 2). Bone scintigraphy revealed no abnormal accumulation of isotope. Blood examination revealed no signs of inflammation or elevation of tumor markers such as carcinoembryonic antigen and alpha-fetoprotein. Cerebrospinal fluid examination revealed no abnormal findings. The preoperative differential diagnosis included meningioma, neurinoma, and migrated disc.

Two months after the onset, the mass was approached by left hemilaminectomy at L-3. A yellowish mass was found in the epidural space at the dorsal edge of the L-3 vertebral body. The mass compressed the L-3 nerve root superiorly, and was elastic hard, easily dissected from the dura mater, and encapsulated by connective tissue. After incision of the capsule, whitish yellow tissue was removed from the inside. Histological examination revealed that the mass was an encapsulated disc (Fig. 3). No abnormality was identified at the L3-4 disc space. The final diagnosis was encapsulated migrated disc. After the operation, he was completely relieved from the leg symptoms and back 

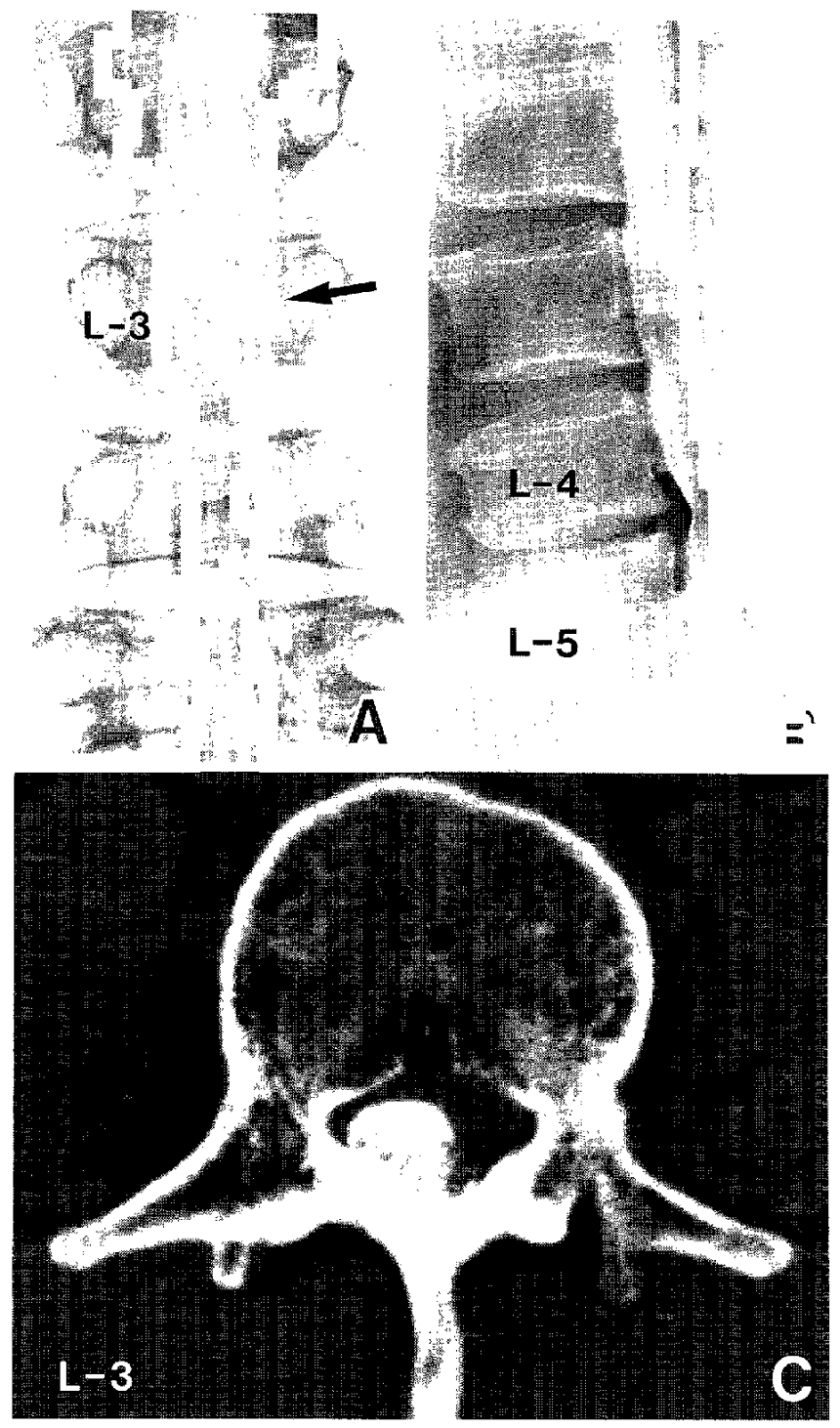

Fig. 1 Myelograms revealing a filling defect at the left side of the L-3 vertebral body (arrow), and mild compression of the subarachnoid space at the L4-5 intervertebral level (A: anteroposterior view, B: lateral view). Computed tomography scan after myelography revealing an intraspinal epidural mass located laterally to the L-3 vertebral body (C).

pain, and returned to work.

\section{Discussion}

The differential diagnosis of a peripherally enhanced epidural mass in the spinal canal includes abscess, tumor with central necrosis, migrated disc, resolving hematoma, and synovial cyst.7) Preoperative discrimination is sometimes difficult. In the present case, the diagnosis of the intraspinal epidural mass as a migrated disc was based on the operative finding and histological analysis. No continuity of the migrated disc with the intervertebral disc and no association of remarkable intervertebral disc degeneration, which made the diagnosis difficult. This fragment was suspected to have originated from the L2-3 intervertebral disc. The symptom started from the L-3 territory and extended to the L-4 territory, so the extruded disc from the L2-3 intervertebral disc probably first compressed the L-3 nerve root, then a part of the disc separated and moved inferiorly and compressed both the L-3 and L-4 nerve roots.

Histological examination of the resected specimen revealed degenerated disc material surrounded by granulation tissue with slight invasion by mononuclear cells. The peripheral enhancement of the migrated disc on MR images was presumably due to the accumulation of contrast agents into the surrounding granulation tissue. ${ }^{2,10)}$ Infiltration of mononuclear cells into the herniated intervertebral disc has been histologically identified previously. ${ }^{1,3,6]}$ These cells, especially macrophages, release chemical mediators and induce neovascularization and persistence of inflammation. ${ }^{1)}$ Both the migration of the sequestered disc and this focal inflammation might be the cause of the aggravation of symptoms during the 2 months between onset and operation. However, small cells infiltrating into the herniated disc may be involved in spontaneous resolution based on histological studies. ${ }^{1,3,6)}$ We cannot deny the possibility that this migrated disc might have been spontaneously absorbed after several months if untreated. ${ }^{4,8]}$

Migrated disc should be included in the differential diagnosis of patients with a long history of back pain and an intraspinal mass. Normal intervertebral disc findings on MR imaging do not exclude a free disc fragment.

\section{References}

1) Doita $M$, Kanatani $T$, Harada $T$, Mizuno $K$ : Immunohistologic study of the ruptured intervertebral disc of the lumbar spine. Spine 21: 235-241, 1996

2) Hirabayashi S, Kumano K, Tsuiki T, Eguchi M, Ikeda $S$ : A dorsally displaced free fragment of lumbar disc herniation and its interesting histologic findings. A case report. Spine 15: 1231-1233, 1990

3) Ito T, Yamada M, Ikuta F, Fukuda T, Hoshi S, Kawaji $Y$, Uchiyama S, Homma T, Takahashi HE: Histologic evidence of absorption of sequestration-type herniated disc. Spine 21: 230-234, 1996

4) Komori $\mathrm{H}$, Shinomiya $\mathrm{K}$, Nakai $\mathrm{O}$, Yamaura I, Takeda S, Furuya K: The natural history of herniated nucleus pulposus with radiculopathy. Spine 21: 225-229, 

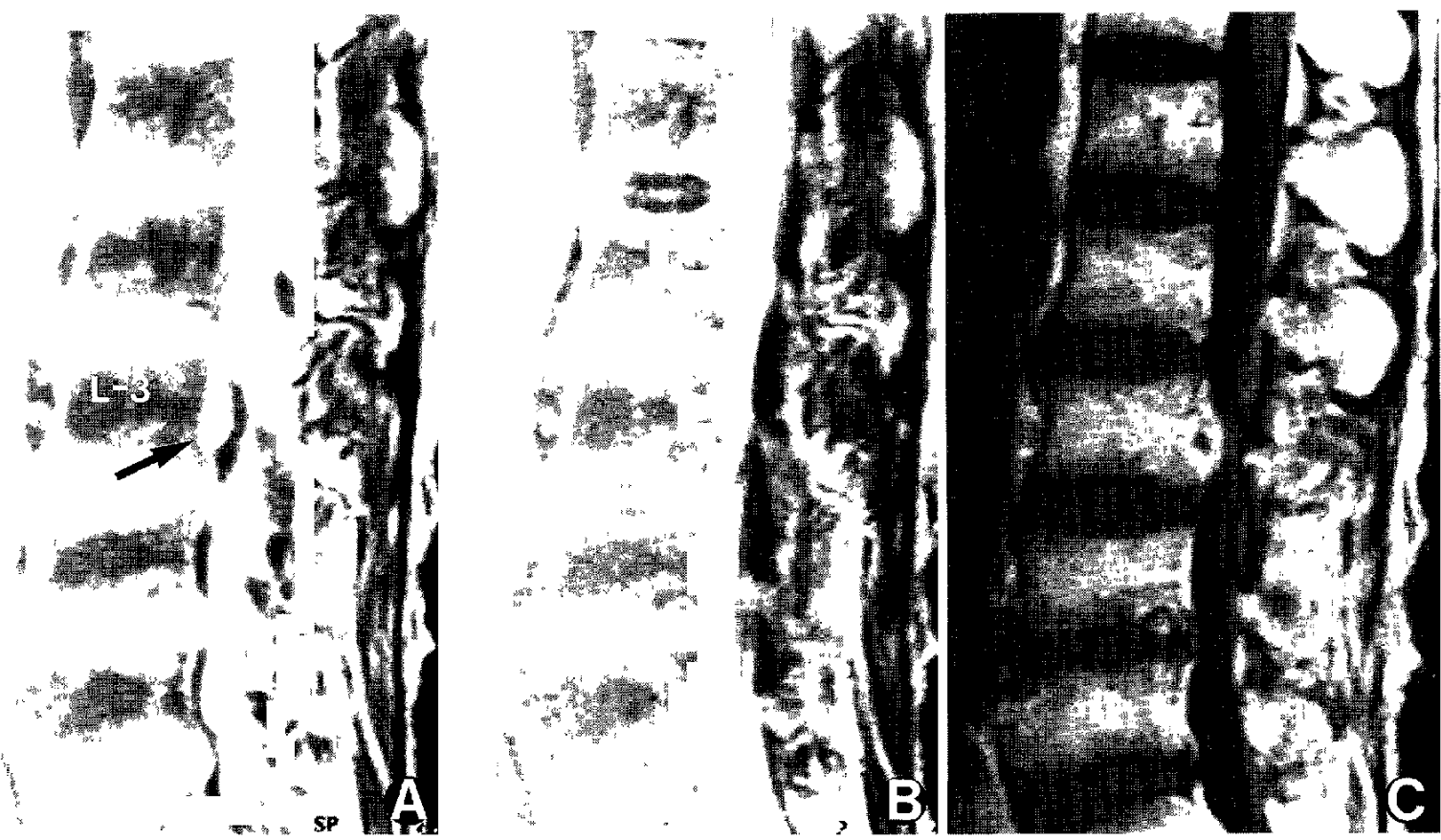

Fig. 2 Magnetic resonance images revealing a low intensity mass on the $T_{1}$-weighted image (A), and a slightly high intensity mass on the $\mathrm{T}_{2}$-weighted image (B) at the dorsal edge of the $\mathrm{L}$-3 vertebral body (arrow). No disc degeneration was observed, except for mild protrusion of the L4-5 intervertebral disc. The mass was peripherally enhanced by the contrast medium (C).

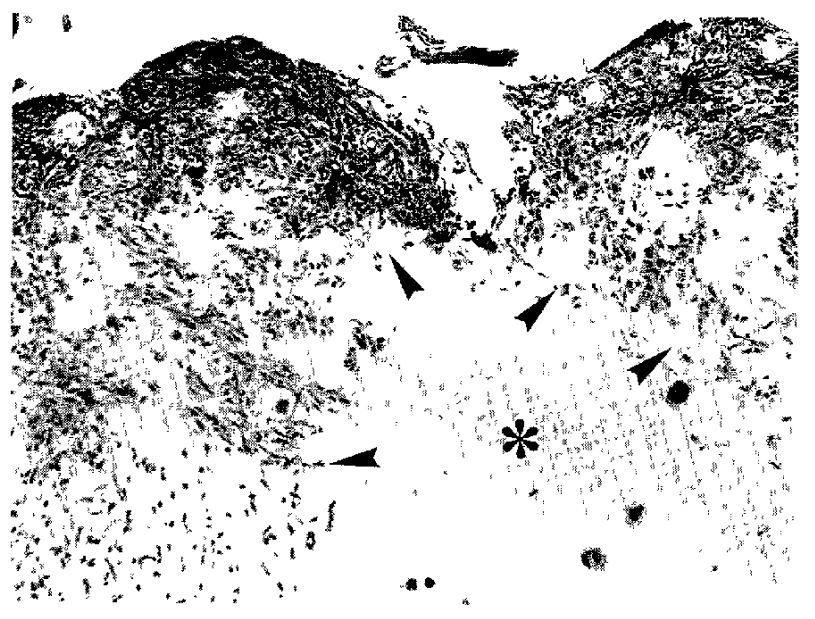

Fig. 3 Photomicrograph showing degenerated disc material (asterisk) surrounded by granulation tissue (arrowheads). HE stain, $\times 100$.
6) Nohara Y, Brown MD, Eurell JC: Lymphatic drainage of epidural space in rabbits. Orthop Clin North Am 22: 189-194, 1991

7) Quint DJ, Colon GP, Chandler WF: Herniated migrated disk fragment with extension into the neural foramen. AJNR Am J Neuroradiol 16: 134, 156, 1995

8) Suzui H, Hanakita J, Suwa H: [Five cases of lumbar disc herniation with apparent radiculopathy showing resolution on follow-up MRI studies]. No Shinkei Geka Journal 7: 671-677, 1998 (Jpn, with Eng abstract)

9) Williams AL, Haughton VM, Daniels DL, Grogan JP: Differential CT diagnosis of extruded nucleus pulposus. Radiology 148: 141-148, 1983

10) Yamashita K, Hiroshima K, Kurata A: GadoliniumDTPA-enhanced magnetic resonance imaging of a sequestered lumbar intervertebral disc and its correlation with pathologic findings. Spine 19: 479-482, 1994
1996

5) Masaryk TJ, Ross JS, Modic MT, Boumphrey F, Bohlman H, Wilber G: High-resolution MR imaging of sequestered lumbar intervertebral discs. AJNR Am J Neuroradiol 9: 351-358, 1988
Address reprint requests to: T. Akai, M.D., Department of Neurosurgery, Kanazawa Medical University, 1-1 Daigaku, Uchinada-machi, Kahoku-gun, Ishikawa 920-0293, Japan. 